\title{
Subleading-colour effects and spin correlations in the PanScales showers
}

\section{Ludovic Scyboz $^{a}$}

${ }^{a}$ Rudolf Peierls Centre for Theoretical Physics, Clarendon Laboratory, Parks Road, University of Oxford, Oxford OX1 3PU, UK

E-mail: ludovic.scyboz@physics.ox.ac.uk

In the context of the PanScales parton shower project, I describe two advances in the development of final-state parton showers with controlled logarithmic accuracy. The first (Ref. [1]) involves simple new algorithms to resolve the long-standing issue of incorrect subleading-colour contributions at leading logarithmic (LL) accuracy in dipole- and antenna-type final-state showers, and also enables the PanScales showers to obtain full-colour NLL accuracy for many observables. The second (Ref. [2]) concerns spin correlations, where we propose a spinor-product based implementation of the well-established Collins algorithm. We verify the logarithmic accuracy of the results with separate purely collinear calculations, using novel spin-sensitive jet observables that are of potential interest also for future experimental measurements.

\footnotetext{
*** The European Physical Society Conference on High Energy Physics (EPS-HEP2021), *** *** 26-30 July 2021 ***

*** Online conference, jointly organized by Universität Hamburg and the research center DESY ***
} 


\section{Introduction}

Most QCD parton showers model the event as an ensemble of colour dipoles which emit additional gluons and quarks, typically under the assumption of a large number of colours, the $N_{C} \rightarrow \infty$ limit. The PanScales family of parton showers introduced recently [3, 4] was shown to achieve next-to-leading-logarithmic (NLL) accuracy for a wide variety of observables at leading colour. The two missing ingredients for claiming full NLL accuracy, at that point, were subleadingcolour corrections, and spin correlations. Below, we introduce two schemes that guarantee the generation of the correct subleading-colour terms at leading-logarithmic accuracy (and at NLL for the PanScales showers, for all but one class of observables). We then present an adapted version of the familiar Collins-Knowles algorithm [5,6] for spin correlations, to be used within dipole and antenna showers. In both cases we validated our implementation by comparing the results to fixed-order matrix elements in the appropriate limits, and at all orders we show below comparisons to known NLL-accurate resummation results.

\section{Subleading-colour effects in the PanScales showers and beyond}

We propose here two algorithms that produce the correct subleading-colour terms at a given logarithmic order. Standard dipole showers usually incorporate part of the finite- $N_{C}$ structure by assigning a colour factor to an emission, depending on whether the identified emitter is a quark $\left(C_{F}\right)$ or a gluon $\left(C_{A} / 2\right)$. It was shown in Ref. [3] that this leads to incorrect contributions at finite- $N_{C}$, already at LL accuracy for certain observables, like the thrust $1-T$. This colour factor assignment is referred to as colour-factor-from-emitter (CFFE scheme) in the following.

We first note that to restore the correct subleading-colour structure at LL accuracy, one needs only consider configurations with emissions that are well-separated in rapidity. In that case, the colour factor assignment is dictated by angular ordering. ${ }^{1}$ Inspired by this argument, we propose an algorithm (dubbed the segment scheme), which essentially keeps track of opening angles as the shower evolves. The second scheme we put forward involves an additional acceptance factor in the shower, which consists of a subleading-colour matrix-element correction (called nested-ordereddouble-soft, or NODS scheme) that reproduces the correct full-colour structure for configurations where a pair of energy-ordered soft gluons, which can be at commensurate rapidites, is wellseparated from all other emissions, with the latter also well-separated from each other in rapidity. The details of the algorithms can be found in the original publication [1].

To validate our approach, and isolate terms at a given logarithmic order, we run the parton showers in a limit where $\alpha_{s} \rightarrow 0$, keeping $\lambda=\alpha_{s} L=-\frac{1}{2}$ fixed. We consider, for an exponentiating event observable $V\left(\alpha_{s}, L\right)$, the quantity

$$
\delta \ln V_{\mathrm{N}^{k} \mathrm{LL}}=\lim _{\alpha_{s} \rightarrow 0}\left(\frac{\ln V_{\mathrm{PS}}\left(\alpha_{s}, \lambda / \alpha_{s}\right)-\ln V_{\mathrm{N}^{k} \mathrm{LL}}\left(\alpha_{s}, \lambda / \alpha_{s}\right)}{\alpha_{s}^{k-1}}\right) .
$$

For a parton shower that is accurate at $\mathrm{N}^{k} \mathrm{LL}$ for the observable $V$, the quantity $\delta \ln V_{\mathrm{N}^{k} L \mathrm{~L}}$ in Eq. (1) vanishes. We show the results of this type of test at LL in Fig. 1a, for a collection of event-shape observables and parton showers (our custom implementation of the Pythia8 shower algorithm [9],

\footnotetext{
${ }^{1}$ The argument was made long ago [7] and is applied, in similar fashion to the algorithm presented below, in Ref. [8].
} 

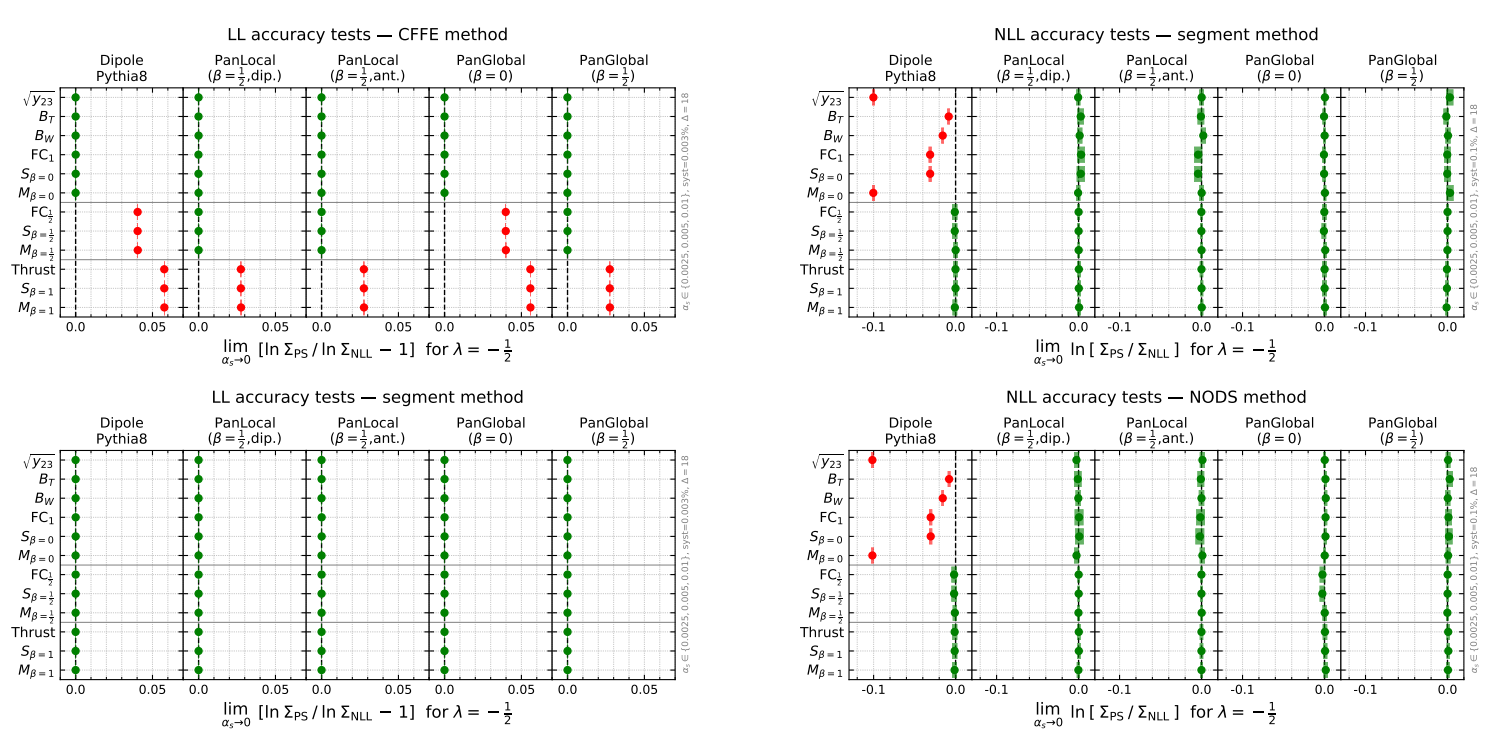

(a) LL tests of global event shapes for the CFFE (top) and segment schemes (bottom), the latter of which solves the

(b) NLL test of the segment (top) and NODS schemes (bottom), which show agreement at full colour for the PanScales showers.

and the PanScales showers). Whereas the CFFE scheme (top) gives the wrong LL subleading-colour terms for some observables, the discrepancies are lifted for all combinations of observables and parton showers with the segment scheme (bottom). Results with the NODS scheme are qualitatively identical. For the subset of parton showers that were NLL-accurate at leading colour (i.e. all showers displayed in the plots except for Pythia8), the segment and NODS schemes reproduce the correct full-colour NLL event shapes, as demonstrated by the consistency tests shown in Fig. 1b.

Neither of the two new schemes is expected to be able to generate the correct subleading-colour terms for non-global observables, which are sensitive to any number of soft, wide-angle gluon emissions (in the single-logarithmic tower, the segment scheme gives the correct first-order $\alpha_{s} L$ term, while the NODS scheme reproduces colour-suppressed terms up to order $\alpha_{s}^{2} L^{2}$ ). Nonetheless, we found good agreement (within $1-2 \%$ over the range we probed) with the finite- $N_{C}$ result computed numerically by Hatta and Ueda for the energy flow in a central slice [10], see section 7.3 of Ref. [1].

\section{Spin correlations in the PanScales showers}

The other essential ingredient that was missing in the PanScales showers for an NLL, leadingcolour accuracy claim is spin correlations. As a matter of fact, an efficient algorithm to include spin correlations in MC event generators has been proposed long ago by Collins [5], and Knowles [6]. ${ }^{2}$

The approach we take is based on the original Collins-Knowles algorithm formulated in the language of spinor products. Here, for a splitting $a \rightarrow b c$ (with helicities $\lambda_{a}, \lambda_{b}$ and $\lambda_{c}$ ), we can write the branching amplitude as

$$
\mathcal{M}_{a \rightarrow b c}^{\lambda_{a} \lambda_{b} \lambda_{c}}=\frac{1}{\sqrt{2}} \frac{g_{s}}{p_{b} \cdot p_{c}} \mathcal{F}_{a \rightarrow b c}^{\lambda_{a} \lambda_{b} \lambda_{c}}(z) S_{\tau}\left(p_{b}, p_{c}\right),
$$

${ }^{2}$ It has been implemented in the context of the Herwig dipole shower [11]. 


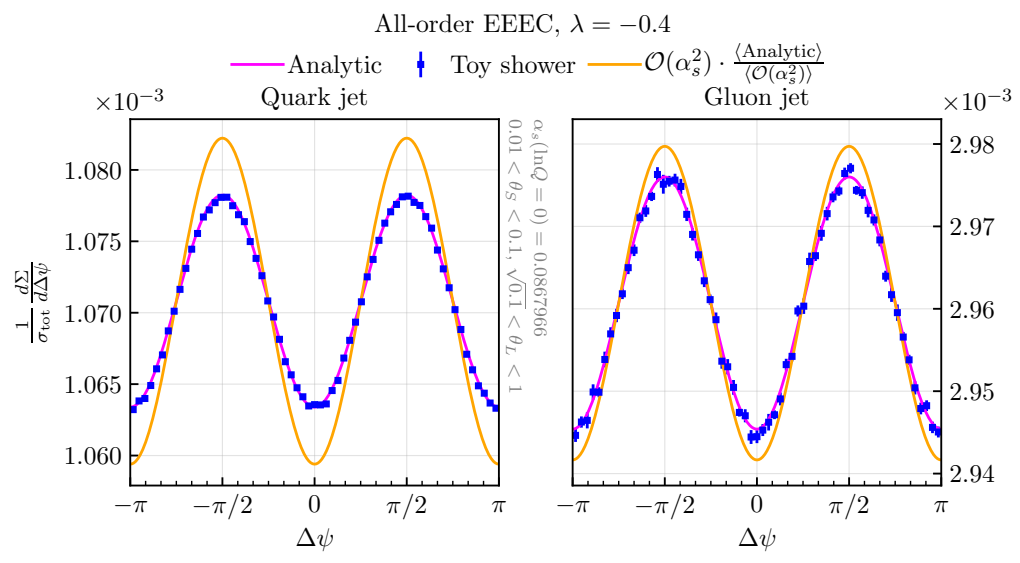

Figure 2: The resummed 3-point energy-energy correlator (EEEC) at $\lambda=-0.4$, with the analytical result from Ref. [14], our toy shower, and the fixed-order expansion rescaled to the mean of the resummed curve. This serves as validation of our toy shower code.

with helicity-dependent Altarelli-Parisi splitting functions $\mathcal{F}_{a \rightarrow b c}^{\lambda_{a} \lambda_{b} \lambda_{c}}(z)$, with $z$ the momentum fraction carried away by parton $b$, and the spinor product $S_{\tau}\left(p_{b}, p_{c}\right)$ (see Appendix A of Ref. [2] for details on the spinor product notation). Equipped with Eq. (2), we can directly apply the CollinsKnowles algorithm to distribute the values of the azimuthal angles in the splittings performed by the parton shower. For the details of the algorithm, please refer to Ref. [2].

In order to validate the implementation of spin correlations in successive collinear splittings, we augmented the MicroJETs code [12,13], which originally serves as a framework for resumming collinear logarithms in small- $R$ jets, with the standard Collins-Knowles algorithm. We tested this numerical setup (which we call the toy shower below) by comparing it to a recent analytical resummation of the 3-point energy-energy correlator (EEEC) performed by Chen, Moult and Zhu [14]. Fig. 2 shows results for the analytic resummation, the toy shower, and the fixed-order result at $O\left(\alpha_{s}^{2}\right)$ rescaled to the mean of the resummed curve for comparison.

Furthermore, we introduce the definition of a new class of Lund-plane inspired observables, which constitute a natural way of using jet substructure information to bring the spin correlations to light. We reconstruct the Lund jet plane [15] by declustering the jets with Cambridge/Aachen [16], and apply the following, IR-safe procedure:

1. Among the list of primary declusterings, choose the highest $k_{t}$ declustering $i \rightarrow j k$ satisfying a cut on the energy fraction $z_{1}>z_{1 \text {, cut }}$,

2. Following the softer branch of that identified primary declustering, choose among the list of secondary declusterings the highest- $k_{t}$ branching $\ell \rightarrow m n$ that satisfies a cut $z_{2}>z_{2}$,cut,

3. Compute the azimuthal angle $\Delta \psi_{12}$ between the primary plane spanned by $\left(p_{j}, p_{k}\right)$ and the secondary plane spanned by $\left(p_{m}, p_{n}\right)$.

In order to isolate the single-logarithimc terms $\alpha_{s}^{n} L^{n}$ generated by the PanScales showers, we run in a limit where the strong coupling is small, $\alpha_{s}=10^{-7}$, but where we keep $\lambda=\alpha_{s} L=-0.5$ fixed. For values of the cuts $z_{1 \text {, cut }}=z_{2 \text {, cut }}=0.1$, we show in Fig. 3 results from the PanLocal (dipole 


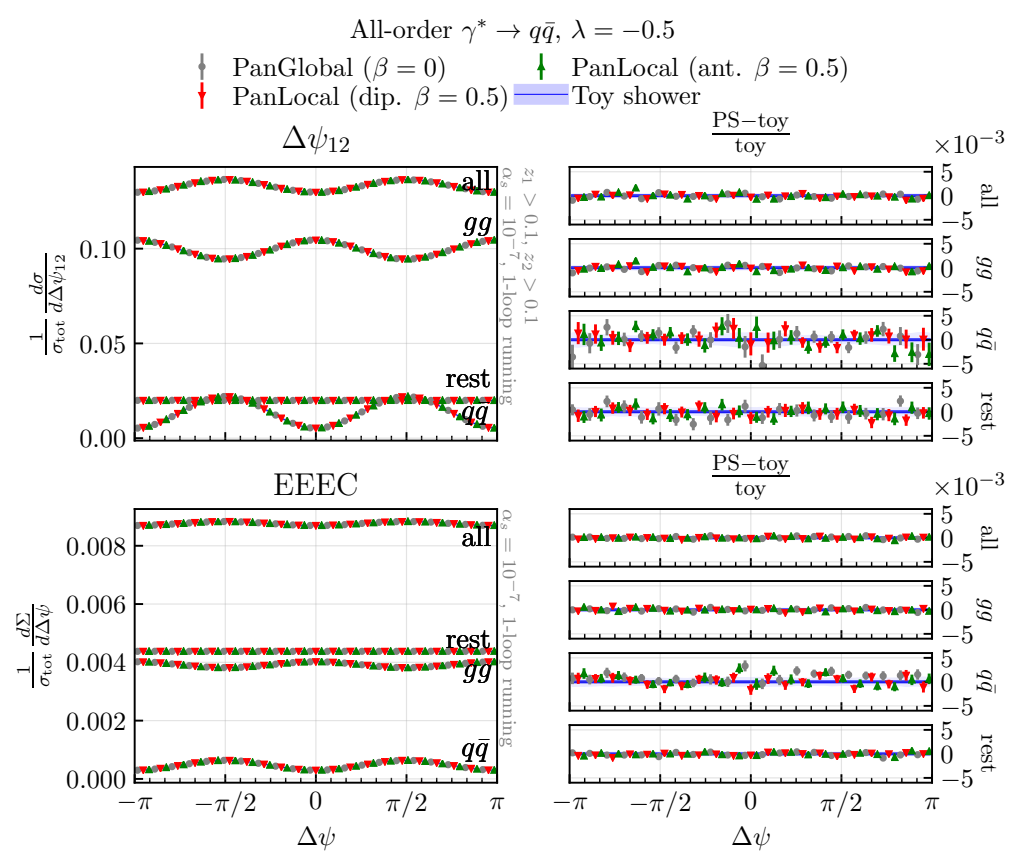

Figure 3: Comparison of the toy shower to three PanScales showers, run in a limit of small $\alpha_{s}=10^{-7}$, for our $\Delta \psi_{12}$ observable and the EEEC. Contributions are separated by flavour channels for the secondary splitting ( $g \rightarrow g g, g \rightarrow q \bar{q}$, rest channel, and all channels summed).

and antenna variants) and the PanGlobal showers, compared to the toy shower for both our $\Delta \psi_{12}$ observable and the EEEC. Spin correlations induce a $\cos (2 \Delta \psi)$ modulation of the difference in azimuthal angles, of opposite signs for the secondary splittings $g \rightarrow g g$ and $g \rightarrow q \bar{q}$. As can be seen, all parton showers agree with the numerical resummation. Phenomenologically, we observe that the azimuthal modulation induced by spin correlations is larger in the $\Delta \psi_{12}$ observable (where the values of the cuts, $z_{1 / 2 \text {,cut }}$ can be further optimised) than in the EEEC: we note that this is of potential interest for future measurements, e.g. in dijet events at the LHC.

\section{Conclusion}

We have introduced two new, efficient algorithms that solve the issue of missing subleadingcolour effects in dipole showers: a segment-based scheme, and a matrix-element correction scheme. These algorithms can be adapted to use in parton showers beyond the PanScales family. We have demonstrated that this implementation gives the correct full-colour result at LL — and at NLL for the PanScales family of showers, for global event shapes (as well as subjet multiplicities, and excellent agreement for a non-global observable). Furthermore, we have presented an adaptation of the Collins-Knowles algorithm for spin correlations, which makes use of a spinor-product formalism. We have proposed a new class of observables, based on the Lund jet plane picture, which can be of phenomenological relevance. Here too, we explicitly validated the implementation of our algorithm at NLL, for two observables sensitive to spin correlations in collinear splittings - the EEEC, and our $\Delta \psi_{12}$ observable. The last remaining class of terms, which need to be captured in order to claim 
complete NLL accuracy for a final-state massless parton shower, is associated with spin correlations in interleaved soft, wide-angle gluon emissions followed by a collinear splitting.

\section{References}

[1] K. Hamilton, R. Medves, G. P. Salam, L. Scyboz and G. Soyez, Colour and logarithmic accuracy in final-state parton showers, JHEP 03 (2021) 041, [2011. 10054].

[2] A. Karlberg, G. P. Salam, L. Scyboz and R. Verheyen, Spin correlations in final-state parton showers and jet observables, Eur. Phys. J. C 81 (2021) 681, [2103 . 16526].

[3] M. Dasgupta, F. A. Dreyer, K. Hamilton, P. F. Monni and G. P. Salam, Logarithmic accuracy of parton showers: a fixed-order study, JHEP 09 (2018) 033, [1805 . 09327].

[4] M. Dasgupta, F. A. Dreyer, K. Hamilton, P. F. Monni, G. P. Salam and G. Soyez, Parton showers beyond leading logarithmic accuracy, Phys. Rev. Lett. 125 (2020) 052002, [2002.11114].

[5] J. C. Collins, Spin Correlations in Monte Carlo Event Generators, Nucl. Phys. B304 (1988) 794-804.

[6] I. Knowles, Angular Correlations in QCD, Nucl. Phys. B 304 (1988) 767-793.

[7] G. Gustafson, Multiplicity distributions in QCD cascades, Nucl. Phys. B392 (1993) 251-280.

[8] J. Holguin, J. R. Forshaw and S. Plätzer, Improvements on dipole shower colour, Eur. Phys. J. C 81 (2021) 364, [2011.15087].

[9] T. Sjöstrand, S. Ask, J. R. Christiansen, R. Corke, N. Desai, P. Ilten et al., An Introduction to PYTHIA 8.2, Comput. Phys. Commun. 191 (2015) 159-177, [1410.3012].

[10] Y. Hatta and T. Ueda, Resummation of non-global logarithms at finite $N_{c}$, Nucl. Phys. B874 (2013) 808-820, [1304.6930].

[11] P. Richardson and S. Webster, Spin Correlations in Parton Shower Simulations, Eur. Phys. J. C80 (2020) 83, [1807.01955].

[12] M. Dasgupta, F. Dreyer, G. P. Salam and G. Soyez, Small-radius jets to all orders in QCD, JHEP 04 (2015) 039, [1411.5182].

[13] M. Dasgupta, F. A. Dreyer, G. P. Salam and G. Soyez, Inclusive jet spectrum for small-radius jets, JHEP 06 (2016) 057, [1602.01110].

[14] H. Chen, I. Moult and H. X. Zhu, Quantum Interference in Jet Substructure from Spinning Gluons, Phys. Rev. Lett. 126 (2021) 112003, [2011.02492].

[15] F. A. Dreyer, G. P. Salam and G. Soyez, The Lund Jet Plane, JHEP 12 (2018) 064, [1807.04758].

[16] Y. L. Dokshitzer, G. D. Leder, S. Moretti and B. R. Webber, Better jet clustering algorithms, JHEP 08 (1997) 001, [hep-ph/9707323]. 